\title{
Author Correction: Crystal structure and optical properties of a high-density InGaN nanoumbrella array as a white light source without phosphors
}

Tetsuya Kouno, Masaru Sakai, Katsumi Kishino, Akihiko Kikuchi, Naoki Umehara and Kazuhiko Hara

Correction to: NPG Asia Materials

https://doi.org/10.1038/am.2016.99

published online 22 July 2016

In Results and discussion, '..., frequency doubled $532 \mathrm{~nm}$ YAG laser...' should read '..., frequency doubled $532 \mathrm{~nm}$ laser...'.

The authors had misrecognized one of the product specifications of the laser used for the experiments. However, it has no effects on the experimental results, discussions, and conclusions. The authors apologize for any inconvenience caused.

The original article has been corrected.

Published online: 1 April 2021 